\title{
THE DETERMINATION OF MODE SHAPES FROM MODERN CROSS-SPECTRAL ESTIMATES
}

\author{
C.Y. LiOU AND T.S. JeNG \\ Department of Computer Science and Information Engineering, National Taiwan University, \\ Taipei, Taiwan, 10764, Republic of China
}

(Received October 1988, accepted November 1988)

In this work we employ three versions of the maximum entropy method (MEM) and a version of the maximum likelihood method (MLM) to estimate the transfer functions among several places of a vibrating structure and use these transfer functions to resolve the modal shapes of the structure. Many conventional methods fail when the structure is undergoing random vibration, or their performance is impaired by the low resolution of the methods or non-stationary nature of the vibration. The modern cross-spectral estimates by the MEM have been proved to be a useful method for random vibration. The important contribution of this work is that, by introducing the maximum likelihood cross-spectral estimate and comparing with those modern cross-spectral estimates by MEM, the mode shapes can be estimated with fairly good results under severe conditions. We test this idea with computer simulations and their results are consistent with our predictions.

\section{INTRODUCTION}

Random vibration problems are important in the dynamics of structures and machines. The natural frequencies and modal shapes are the most significant parameters concerning the condition of the structures or the machines. There are many existing methods to estimate these parameters. The spectral approaches of these parameters are used frequently in estimating the transfer functions at different positions of a structure and obtaining its mode shapes. We now briefly review the conventional spectral estimations (see [1] for details). Conventional spectral estimations are based on the Fourier transform of finite data and take advantage of the fast Fourier transform (FFT) to speed comutation. These methods suffer a tradeoff between resolution and variance because of the finite amount of data, and cannot resolve the mode shapes under severe environmental conditions. Burke et al. in 1980 proposed a different method for the problem of estimating mode shapes in ocean environments $[2,3]$. In order to improve the performance in both resolution and variance, Briggs et al. [4] in 1982 proposed an approach which applied the modern cross-spectral estimates to improve the estimated transfer functions and improve the mode shape estimation. Briggs concluded that the transfer function estimated by the cross-spectrum is better than by the autospectrum. Briggs' method takes advantage of the high resolution ability of the multichannel maximum entropy method (MEM) for the recorded finite data [5-7]. There are many varieties of MEM; we will employ three famous algorithms-the Yule-Walker, Burg, and Morf algorithms. This choice is based on our own experiences, and are well established. Liou in 1985 derived another method of multichannel cross-spectral estimates under the name of multichannel maximum likelihood method (MLM) [8]. This method can be derived in a different way $[9,10]$. The MLM is best known for its simple appearance, stability, and resolution. We are interested in comparing its ability in mode shape estimation with the MEMs. 
In section 2, we briefly review the estimation of transfer functions and cross-spectral estimates by the multichannel MEM and MLM. In section 3, we compare the performance of MEM and MLM in the estimation of mode shapes using both a lumped mass system and a continuous mass system. In section 4 , we provide a summary of this work.

\section{TRANSFER FUNCTIONS AND CROSS-SPECTRAL ESTIMATES}

In this work we will estimate the transfer functions between every two positions among several specified positions in a giving structure. The amplitude and phase relation of the transfer function between the two positions are used as the mode shape relations. We will further assume the modes can be well discriminated in the frequency domain by using the modern spectral analyses. The transfer function estimates [1] between the position $i$ and $l$ is defined as following:

$$
\begin{gathered}
H_{i l}(f)=\frac{S_{i l}(f)}{S_{i i}(f)}, \quad 0 \leqslant f<\infty, \\
H_{i l}(f)=\left|H_{i l}\right|(f) \mathrm{e}^{-j \phi_{i l}(f)}, \\
\left|H_{i l}(f)\right|=\frac{\left|S_{i l}(f)\right|}{S_{i i}(f)} \quad 0 \leqslant f<\infty,
\end{gathered}
$$

where $S_{i l}(f)$ and $S_{i i}(f)$ are the estimated cross-spectrum and autospectrum by the MEM or MLM. The phase spectrum, $\phi_{i l}(f)$, can be obtained from equation (2) by factorising the phase term of the complex valued transfer function, $H_{i j}(f)$. The coherence spectrum is defined as following:

$$
\gamma_{i l}(f)=\frac{\left|S_{i l}(f)\right|^{2}}{S_{i i}(f) S_{l l}(f)} .
$$

In our simulation cases the phase $\phi(f)$ is close to $0^{\circ}$ or $180^{\circ}, \gamma_{i l}(f)$ is close to 1 , and there is a significant (large) peak in the magnitude cross-spectrum graph $\left|S_{i l}(f) 0 \leqslant f<\infty\right|$ at any natural frequency, $f_{n}$. These relations are useful for the identification of the natural frequencies $f_{n}$ of the structure. Once a natural frequency $f_{n}$ has been found, the transfer functions at frequency $f_{n}$ can be estimated by substituting the estimated cross-spectral $S_{i l}\left(f_{n}\right)$ and autospectral $S_{i i}\left(f_{n}\right)$ in equation (1) to obtain $H_{i l}\left(f_{n}\right)$. Then the estimated magnitude vector and phase vector of a mode shape is given by

$$
\begin{gathered}
\mathbf{u}\left(f_{n}\right)=\left[\left|H_{11}\left(f_{n}\right)\right|,\left|H_{12}\left(f_{n}\right)\right|,\left|H_{13}\left(f_{n}\right)\right|, \ldots,\left|H_{1 l}\left(f_{n}\right)\right|, \ldots\right]^{\mathrm{T}}, \\
\boldsymbol{\phi}\left(f_{n}\right)=\left[\phi_{11}\left(f_{n}\right), \phi_{12}\left(f_{n}\right), \phi_{13}\left(f_{n}\right), \ldots, \phi_{1 l}\left(f_{n}\right), \ldots\right]^{\mathrm{T}} .
\end{gathered}
$$

We briefly review the multichannel (two channels in this work) MLM and MEM algorithms in the following paragraphs. Let

$$
\mathbf{x}_{i l}=\left[x_{i}\left(t_{1}\right), x_{l}\left(t_{1}\right), x_{i}\left(t_{2}\right), x_{l}\left(t_{2}\right), x_{i}\left(t_{3}\right), x_{l}\left(t_{3}\right), \ldots, x_{i}\left(t_{M+1}\right), x_{l}\left(t_{M+1}\right)\right]^{\mathrm{T}},
$$

where $x_{i}\left(t_{m}\right)$ is the measured acceleration or displacement at position $i$ at time $t_{m}=m \cdot \Delta$ and $\mathbf{x}$ is a column vector with dimension $2(M+1) . \Delta$ is the sampling interval. The MLM cross-spectral estimate is defined as:

$$
\left[S_{i l}(f)\right]=\left[G^{\dagger} R_{i l}^{-1} G\right]^{-1},
$$


where

$$
\begin{aligned}
& G=\left(\begin{array}{ccccccccc}
\mathrm{e}^{j 2 \pi f t_{1}} & 0 & \mathrm{e}^{j 2 \pi f t_{2}} & 0 & \mathrm{e}^{j 2 \pi f t_{3}} & 0 & \cdots & \mathrm{e}^{j 2 \pi f t_{M+1}} & 0 \\
0 & \mathrm{e}^{j 2 \pi f t_{1}} & 0 & \mathrm{e}^{j 2 \pi f t_{2}} & 0 & \mathrm{e}^{j 2 \pi f t_{3}} & \cdots & 0 & \mathrm{e}^{j 2 \pi f t_{M+1}}
\end{array}\right)^{\mathrm{T}}, \\
& R_{i l}=\left(\begin{array}{ccccc}
R_{i l}(0) & R_{i l}(-1) & R_{i l}(-2) & \cdots & R_{i l}(-M) \\
R_{i l}(1) & R_{i l}(0) & R_{i l}(-1) & \cdots & R_{i l}(-M+1) \\
R_{i l}(2) & R_{i l}(1) & R_{i l}(0) & \cdots & R_{i l}(-M+2) \\
\vdots & \vdots & \vdots & \ddots & \vdots \\
R_{i l}(M) & R_{i l}(M-1) & R_{i l}(M-2) & \cdots & R_{i l}(0)
\end{array}\right)
\end{aligned}
$$

with each submatrix

$$
R_{i l}(n)=\left(\begin{array}{ll}
r_{i i}(n) & r_{i l}(n) \\
r_{l i}(n) & r_{l l}(n)
\end{array}\right) .
$$

In the above formula, $G^{\dagger}$ denotes the Hermitian matrix of the $2(M+1) \times 2$ matrix $G$, and $M+1$ is the filter order. The $2(M+1) \times 2(M+1)$ matrix $R_{i l}$ is equal to the expectation of the matrix $\left[\mathbf{x}_{i l} \mathbf{x}_{i l}^{\mathrm{T}}\right]$ and each entry $\gamma_{i l}(n)$ in $R_{i l}(n)$ is equal to the expectation value (correlation) of $x_{i}\left(t_{k+n}\right) x_{l}\left(t_{k}\right)$. An estimated correlation

$$
\hat{r}_{i l}(n)-\frac{1}{N} \sum_{m=1}^{N} x_{i}\left(t_{m+n}\right) x_{l}\left(t_{m}\right)
$$

or other methods $[1,11]$ will replace the theoretical value $r_{i l}(n)$ in equation $(4)$. In equation (4) $\left[S_{i l}(f)\right]$ is a complex $2 \times 2$ square matrix and

$$
\left[S_{i l}(f)\right]=\left(\begin{array}{cc}
\hat{S}_{i i}(f) & \hat{S}_{i l}(f) \\
\hat{S}_{l i}(f) & \hat{S}_{l l}(f)
\end{array}\right)
$$

where $\hat{S}_{i l}(f)$ is the estimated cross-spectral estimate at frequency $f$ using estimated correlations $\hat{r}_{i l}(n)$. From now on we will omit the hat ${ }^{\wedge}$ in all $\hat{\boldsymbol{S}}$ for convenience.

Since MEM is well known and well established, we briefly review its algorithms for later use. Let $\mathbf{x}_{i l}(m)=\left[x_{i}\left(t_{m}\right), x_{l}\left(t_{m}\right)\right]^{\mathrm{T}}$ be a two-dimensional column vector. Two prediction error (PE) vectors $\mathbf{E}_{M, n}^{f}$ and $\mathbf{E}_{M, n}^{b}$, which are two-dimensional column vectors, are defined for the data $\mathbf{x}_{i l}(m)$. These are

$$
\begin{gathered}
\mathbf{E}_{M, n}^{f}=\sum_{m=0}^{M} A_{M, m} \mathbf{x}_{i l}(n-m), \\
\boldsymbol{E}_{M, n}^{b}=\sum_{m=0}^{M} B_{M, M-m} \mathbf{x}_{i l}(n-m),
\end{gathered}
$$

where $A_{M, n}$, and $B_{M, n}$ are forward and backward autoregression (AR) filter coefficients respectively. The filter order is $M+1$. The expectation energies of the two PE matrices are defined by

$$
\begin{aligned}
& P_{M}^{f}=E\left[\left(\mathbf{E}_{M, n}^{f}\right)\left(\mathbf{E}_{M, n}^{f}\right)^{\dagger}\right] \\
& P_{M}^{b}=E\left[\left(\mathbf{E}_{M, n}^{b}\right)\left(\mathbf{E}_{M, n}^{b}\right)^{\dagger}\right] .
\end{aligned}
$$

By minimizing the forward and backward expectation PE energy, we obtain the matrix 
equation for the backward and forward AR filters.

$$
\left(\begin{array}{cccc}
R_{i l}(0) & R_{i l}(-1) & \cdots & R_{i l}(-M) \\
R_{i l}(1) & R_{i l}(0) & \cdots & R_{i l}(1-M) \\
\vdots & \vdots & \ddots & \vdots \\
R_{i l}(M) & R_{i l}(M-1) & \cdots & R_{i l}(0)
\end{array}\right)\left(\begin{array}{cc}
I & B_{M, M} \\
A_{M, 1} & B_{M, M-1} \\
\vdots & \vdots \\
A_{M, M-1} & B_{M, 1} \\
A_{M, M} & I
\end{array}\right)=\left(\begin{array}{cc}
P_{M}^{\prime} & 0 \\
0 & 0 \\
\vdots & \vdots \\
0 & 0 \\
0 & P_{M}^{b}
\end{array}\right)
$$

where $R_{i l}(n)=E\left\{\mathbf{x}_{i l}(t+n) \mathbf{x}_{i l}(t)^{T}\right\}$ are a $2 \times 2$ square submatrix. The $Z$-transforms [11] of the two filters are given below.

$$
\begin{gathered}
A_{M}(z)=I+A_{M, 1} z^{-1}+\cdots+A_{M, M} z^{-M} \\
B_{M}(z)=B_{M, M}+B_{M, M-1} z^{-1}+\cdots+I z^{-M}
\end{gathered}
$$

The two-channel MEM spectral density estimates are given by

$$
\begin{gathered}
{\left[S_{i l}^{f}(f)\right]=\sum_{m=-\infty}^{\infty} R_{m} z^{-m}=A_{M}^{-1}(z) P_{M}^{f} A_{M}^{-\dagger}\left(\frac{1}{z^{*}}\right)} \\
{\left[S_{i l}^{b}(f)\right]=B_{M}^{-1}(z) P_{M}^{b} B_{M}^{-\dagger}\left(\frac{1}{z^{*}}\right)}
\end{gathered}
$$

where $\left[S_{i l}^{f}(f)\right]$ and $\left[S_{i l}^{b}(f)\right]$ are complex $2 \times 2$ square matrices and ${ }^{*}$ denotes the complex conjugate. They are

$$
\left[S_{i l}^{f}(f)\right]=\left(\begin{array}{ll}
S_{i i}(f) & S_{i l}(f) \\
S_{l i}(f) & S_{l l}(f)
\end{array}\right) ; \quad\left[S_{i l}^{b}(f)\right]=\left(\begin{array}{ll}
S_{i i}(f) & S_{i l}(f) \\
S_{l i}(f) & S_{l l}(f)
\end{array}\right) .
$$

There are three algorithms to obtain the forward and backward AR filters, $A_{M, n}$ and $B_{M, n}$. They are the Yule-Walker, Burg, and Morf algorithms [5-7]. Burg's algorithm operates directly on the data $x_{i l}(m)$. Both the Yule-Walker algorithm and Morf algorithm use the estimated correlation functions $\hat{r}_{i l}(n)$ explicitly. The Morf algorithm will give a unique AR filter coefficient, $\left[S_{i l}^{b}(f)\right]=\left[S_{i l}^{f}(f)\right]$. In both the Burg and the Yule-Walker algorithm we will use $\left[S_{i l}^{f}(f)\right]$ as the cross-spectral estimate. Once the filter coefficients have been determined, we can calculate the cross-spectra matrix, $[S(f)]$, and then substitute its data in equations (1)-(3) to obtain the transfer functions and the mode shape vector.

\section{APPLICATIONS IN MODAL SHAPE IDENTIFICATION}

In this section we will apply the algorithms in the above section to estimate the modal shapes of two systems. One is a three-degree-of-freedom lumped mass system, the other is a one-dimensional continuous mass system. We now describe the two dynamic systems and their simulations in the following two cases.

CASE A: LUMPED MASS SYSTEM

The first system is a linear one-dimensional lumped mass system consisting of three masses $m_{i}(i=1,2,3)$ connected by springs and dampers. The dynamic equation of this system is

$$
[m]\left\{\frac{\mathrm{d}^{2} \mathbf{y}(t)}{\mathrm{d}^{2} t}\right\}+[c]\left\{\frac{\mathrm{dy}(t)}{\mathrm{d} t}\right\}+[k]\{\mathbf{y}(t)\}=\{\mathbf{n}(t)\}
$$


where

$$
[\dot{m}]=\left(\begin{array}{ccc}
m_{1} & 0 & 0 \\
0 & m_{2} & 0 \\
0 & 0 & m_{3}
\end{array}\right), \quad[k]=\left(\begin{array}{ccc}
k_{1}+k_{2} & -k_{2} & 0 \\
-k_{2} & k_{2}+k_{3} & -k_{3} \\
0 & -k_{3} & k_{3}
\end{array}\right), \quad[c]=\alpha[m]+\beta[k] .
$$

In our case $m_{1}=m_{2}=1, m_{3}=2, k_{1}=k_{2}=30, k_{3}=60, \alpha=\beta=0.006$ and the theoretical undamped three natural frequencies of the above system are $0.325,1.150$ and $1.768 \mathrm{~Hz}$. In the dynamic equation, $y(t)=\left[y_{1}(t), y_{2}(t), y_{3}(t)\right]^{\mathrm{T}}$ denotes the displacement vector of the three masses. $\mathrm{n}(t)=\left[n_{1}(t), n_{2}(t), n_{3}(t)\right]^{\mathrm{T}}$ is the simulated white Gaussian random input forces to the three masses and all $n_{i}(t)(i=1,2,3)$ have independent identical probability distribution. By using the Euler implicit formulas [11], the dynamic differential equation is simulated by a difference equation. The simulated random forces can be generated by computer simulations. The accelerations

$$
x_{i}\left(t_{m}\right)=\frac{\mathrm{d}^{2} y_{i}\left(t_{m}\right)}{\mathrm{d} t^{2}}, \quad i=1,2,3
$$

at the three masses are recorded spontaneously and used for later modal shape estimation. The sampling rate is $10 \mathrm{~Hz}$ and the recorded length is $(102.4 \mathrm{~s})$. A total of 1024 data samples is obtained for each mass by truncating the transient response data to reduce the effect of initial conditions.

The unbiased correlation functions which will be used in the MLM and Yule-Walker algorithm are estimated by a fast Fourier transform (FFT) based algorithm [1, 11]. The techniques of segmenting and averaging the 1024 data in MEM and MLM are properly determined by conventions and some experience to achieve high performance. They are: (1) MEM, Yule-Walker algorithm: four segments of 256 data each. (2) MEM, Burg algorithm: four segments of 256 data each. (3) MEM, Morf algorithm: 16 segments of 64 data each. (4) MLM, 16 segments of 64 data each. Four representative figures are included in Figs 1-4. These four figures are part of the figures for $\left|S_{i l}\right|$, the magnitude cross-spectral between masses $i$ and $l$, obtained by using the given segmenting and averaging to display roughly the relative performance. The natural frequencies can be

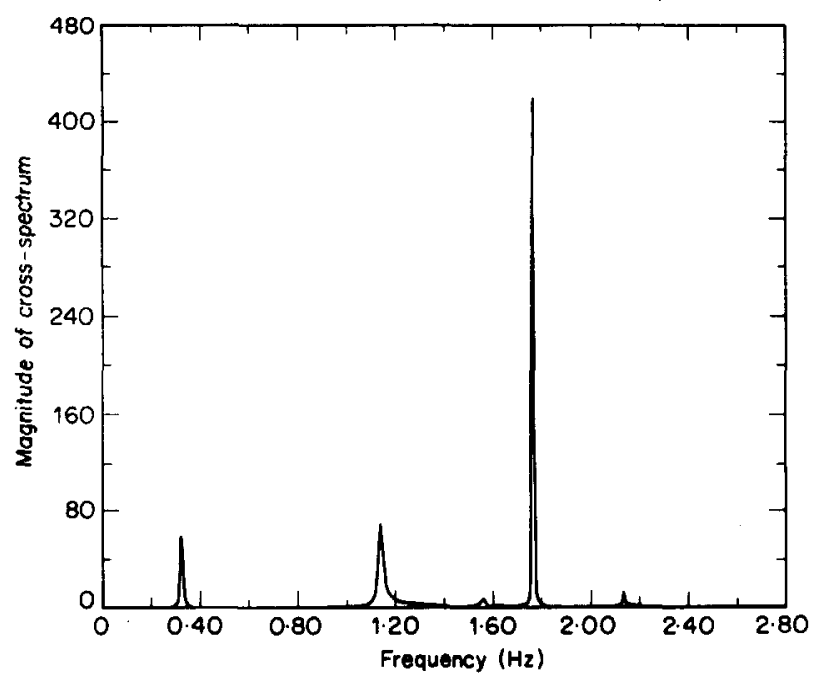

Figure 1. Cross-spectral magnitude by the Yule-Walker algorithm (filter order $=20$ ) for the 3 dof lumped mass system. 


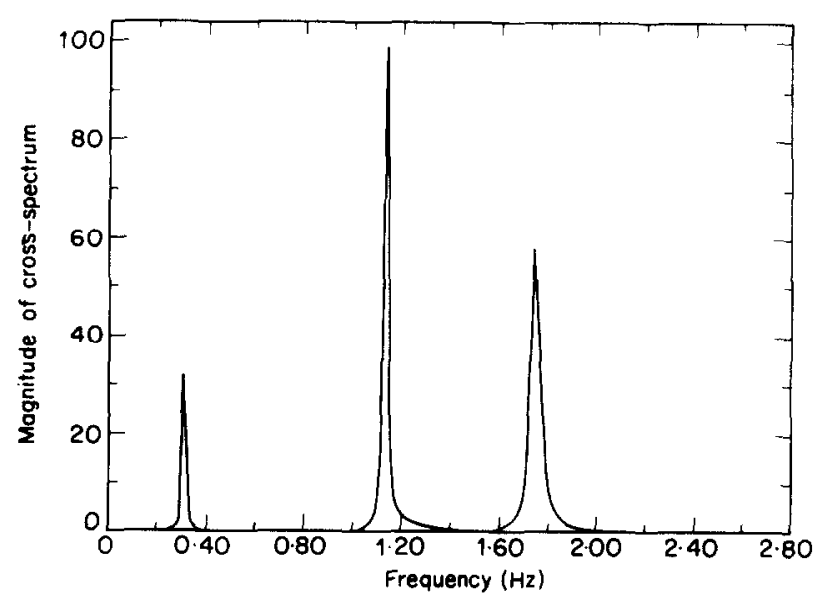

Figure 2. Cross-spectral magnitude by the Burg algorithm (filter order $=20$ ) for the 3 dof lumped mass system.

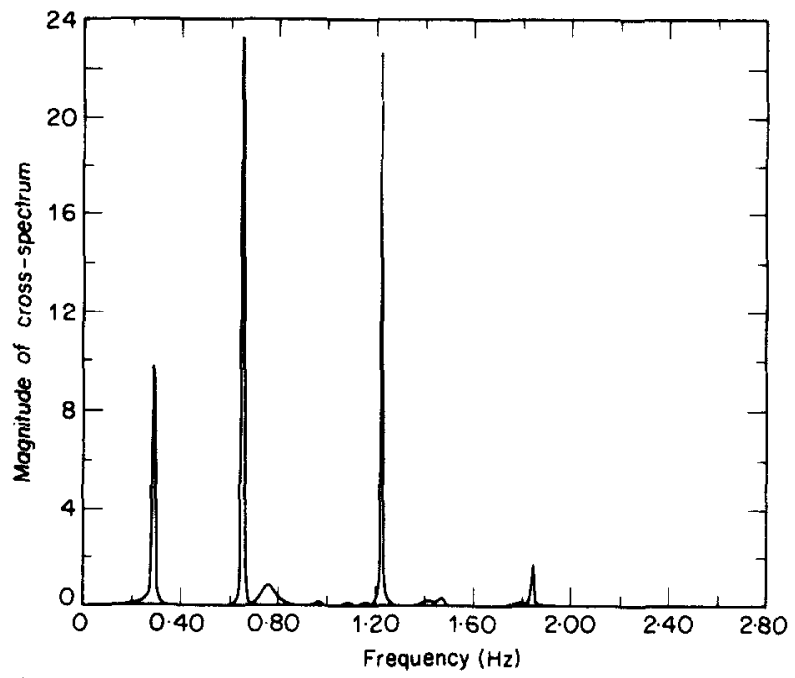

Figure 3. Cross-spectral magnitude by the Morf algorithm (filter order $=13$ ) for the 3 dof lumped mass system.

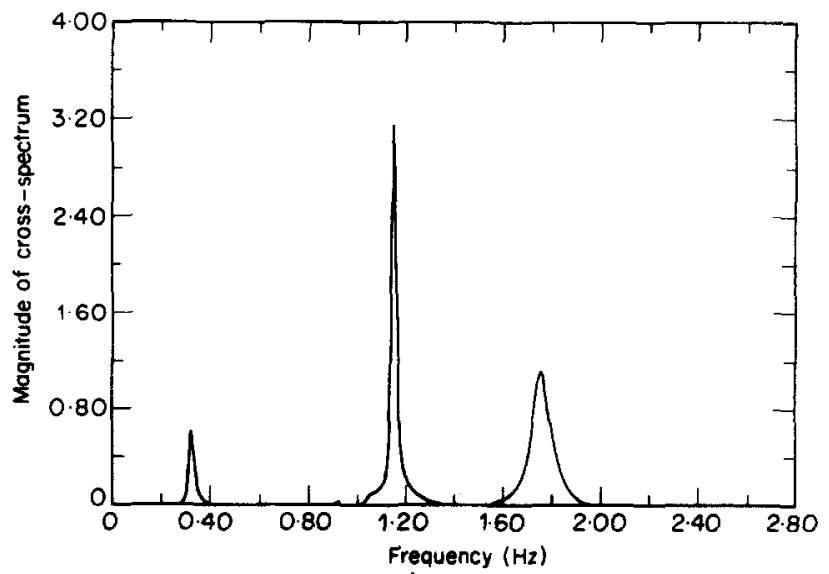

Figure 4. Cross-spectrai magnitude by MLM for the 3 dof lumped mass system. 
easily identified by frequencies below the large peaks in these magnitude cross-spectral graphs. The averaged natural frequencies over all such figures are listed in Table 1 . The undamped natural frequencies are the theoretical values, $[c]=0$. Since the damping factor is very small, the damped natural frequencies are very close to the undamped natural

TABLE 1

\begin{tabular}{lccc}
\hline & \multicolumn{3}{c}{ Frequency } \\
\cline { 2 - 4 } \multicolumn{1}{c}{ Method } & Mode 1 & Mode 2 & Mode 3 \\
\hline Undamped & 0.325 & 1.152 & 1.768 \\
Yule-Walker & 0.322 & 1.143 & 1.768 \\
Burg & 0.313 & 1.143 & 1.748 \\
Morf & 0.293 & 1.221 & 1.836 \\
MLM & 0.322 & 1.152 & 1.758 \\
\hline
\end{tabular}

frequencies. From our experience with the Morf algorithm, we find that Morf's MEM has several disadvantages on modal analyses. We summarise its disadvantages as follows: (1) It is unstable when the filter order $M+1$ changes slightly. (2) Its spectra always have high peaks at non-natural frequencies. This has caused trouble in identifying the natural frequencies. (3) It has a large bias in the natural frequency estimation. Therefore, we will not use the Morf algorithm in the following work. In order to estimate the mode shape vector $\mathbf{u}\left(f_{n}\right)$ and $\phi\left(f_{n}\right)$, we estimate the magnitude cross-spectra $\left|S_{i l}\left(f_{n}\right)\right|$ in the beginning, then substitute these estimated $S_{i l}\left(f_{n}\right)$ in equations (1)-(3) to obtain the transfer functions, $H_{i l}\left(f_{n}\right)$. Using equations (4) and (5), we obtain the estimated mode shapes. We will fix the $i=1$ and change the $l=2,3$. The theoretical mode shapes are shown in Table 2 .

TABLE 2

\begin{tabular}{cccrr}
\hline Mode & Frequency (Hz) & Mass 1 & Mass 2 & \multicolumn{1}{c}{ Mass 3 } \\
\hline 1 & 0.325 & 1.000 & 1.861 & 2.162 \\
2 & 1.152 & 1.000 & 0.254 & -0.341 \\
3 & 1.768 & 1.000 & -2.115 & 0.679 \\
\hline
\end{tabular}

Tables 3-5 are the three mode shapes estimated by using the Yule-Walker and Burg algorithms, and MLM.

\section{CASE B. ONE-DIMENSIONAL LINEAR STRING SYSTEM}

Consider a one-dimensional string with uniform mass density $\rho=1$. It is fixed at $z=0$ and $z=L=100$ with constant tension $T=100$. Its motion is given by the following differential equation:

$$
T \frac{\partial^{2} y(z, t)}{\partial z^{2}}+f(z, t)=\rho \frac{\partial^{2} y(z, t)}{\partial t^{2}} .
$$

The natural frequencies $f_{n}$ and mode shapes are

$$
f_{n}=\frac{n}{2} \sqrt{\frac{T}{\rho L^{2}}} \quad Y_{n}(z)=A_{n} \sin \left(\frac{n \pi z}{L}\right) .
$$


TABLE 3

Yule-Walker's algorithm with filter order $M+1=20$ and $\alpha=\beta=0.006$

\begin{tabular}{|c|c|c|c|}
\hline $\begin{array}{l}\text { Mass } \\
i \& l\end{array}$ & $\begin{array}{c}\text { Phase (degree) } \\
\phi^{0}\left(f_{n}\right)\end{array}$ & $\begin{array}{l}\text { Coherence squared } \\
\qquad \gamma\left(f_{n}\right)\end{array}$ & $\begin{array}{l}\text { Transfer function } \\
\qquad\left|H_{i l}\left(f_{n}\right)\right|\end{array}$ \\
\hline \multicolumn{4}{|c|}{ First mode at $f_{n}=0.322 \mathrm{~Hz}$} \\
\hline $1 \& 2$ & $0 \cdot 0$ & 1.00 & $1 \cdot 859$ \\
\hline $1 \& 3$ & $0 \cdot 1$ & $1 \cdot 00$ & $2 \cdot 162$ \\
\hline \multicolumn{4}{|c|}{ Second mode at $f_{12}=1.143 \mathrm{~Hz}$} \\
\hline $1 \& 2$ & $0 \cdot 3$ & $1 \cdot 00$ & $0 \cdot 253$ \\
\hline $1 \& 3$ & $177 \cdot 0$ & $1 \cdot 00$ & $0 \cdot 334$ \\
\hline \multicolumn{4}{|c|}{ Third mode at $f_{n}=1.768 \mathrm{~Hz}$} \\
\hline $1 \& 2$ & $179 \cdot 2$ & $1 \cdot 00$ & $1 \cdot 990$ \\
\hline $1 \& 3$ & $0 \cdot 6$ & 0.97 & 0.637 \\
\hline
\end{tabular}

TABLE 4

Burg's algorithm with filter order $M+1=20$ and $\alpha=\beta=0.006$

\begin{tabular}{|c|c|c|c|}
\hline $\begin{array}{l}\text { Mass } \\
i \& l\end{array}$ & $\begin{array}{c}\text { Phase (degree) } \\
\phi^{\circ}\left(f_{n}\right)\end{array}$ & $\begin{array}{l}\text { Coherence squared } \\
\qquad \gamma\left(f_{n}\right)\end{array}$ & $\begin{array}{l}\text { Transfer function } \\
\qquad\left|\boldsymbol{H}_{i l}\left(f_{n}\right)\right|\end{array}$ \\
\hline \multicolumn{4}{|c|}{ First mode at $f_{n}=0.313 \mathrm{~Hz}$} \\
\hline $1 \& 2$ & 0.0 & 1.00 & $1 \cdot 830$ \\
\hline $1 \& 3$ & $0 \cdot 4$ & $1 \cdot 00$ & $2 \cdot 187$ \\
\hline \multicolumn{4}{|c|}{ Second mode at $f_{n}=1 \cdot 143 \mathrm{~Hz}$} \\
\hline $1 \& 2$ & 0.9 & 0.99 & $0 \cdot 250$ \\
\hline $1 \& 3$ & $178 \cdot 0$ & $1 \cdot 00$ & $0 \cdot 330$ \\
\hline \multicolumn{4}{|c|}{ Third mode at $f_{n}=1.748 \mathrm{~Hz}$} \\
\hline $1 \& 2$ & $177 \cdot 7$ & 0.97 & $2 \cdot 858$ \\
\hline $1 \& 3$ & $3 \cdot 2$ & 0.97 & 0.578 \\
\hline
\end{tabular}

TABLE 5

$M L M$ with filter order $M+1=20$ and $\alpha=\beta=0.006$

\begin{tabular}{lccc}
\hline $\begin{array}{c}\text { Mass } \\
i \& l\end{array}$ & $\begin{array}{c}\text { Phase (degree) } \\
\phi^{0}\left(f_{n}\right)\end{array}$ & $\begin{array}{c}\text { Coherence squared } \\
\gamma\left(f_{n}\right)\end{array}$ & $\begin{array}{c}\text { Transfer function } \\
\left|H_{i l}\left(f_{n}\right)\right|\end{array}$ \\
\hline \multicolumn{4}{c}{ First mode at $f_{n}=0.322 \mathrm{~Hz}$} \\
$1 \& 2$ & 0.2 & 1.00 & \\
$1 \& 3$ & 0.4 & 1.00 & 1.896 \\
& & Second mode at $f_{n=1}=1.152 \mathrm{~Hz}$ & 2.200 \\
$1 \& 2$ & 0.4 & 0.99 \\
$1 \& 3$ & 178.8 & 1.00 & 0.251 \\
& & Third mode at $f_{n=1}=1.758 \mathrm{~Hz}$ & 0.338 \\
$1 \& 2$ & 179.6 & 0.89 & \\
$1 \& 3$ & 0.1 & 0.94 & 2.030 \\
\hline
\end{tabular}


In order to simulate this string, we apply a finite element method and discretise the string into nine elements with nine nodes (or nine degrees of freedom). The dynamics of the discretized system are obtained as below.

$$
[m]\left(\frac{\mathrm{d}^{2} \mathbf{y}(t)}{\mathrm{d} t^{2}}\right)+[c] \frac{\mathrm{d} \mathbf{y}(t)}{\mathrm{d} t}+[k]\{\mathbf{y}(t)\}=\{\mathbf{n}(t)\} .
$$

Proportional damping is added to the above equation, $[c]=\alpha[m]+\beta[k], \alpha=\beta=0.02$. The simulation of the above differential equation is analogous with the lumped mass system in case A. We recorded the 1024 samples of the accelerations

$$
x_{i}\left(t_{m}\right)=\frac{\mathrm{d}^{2} y_{i}\left(t_{m}\right)}{\mathrm{d} t^{2}}, \quad i=1,2,3, \ldots, 9
$$

for each of the nine nodes. We use the same segmenting and averaging combinations as for the lumped mass system in case 1, and apply the two channel MEM and MLM to obtain the cross-spectra between every two nodes. Three typical magnitude cross-spectra between node 1 and node 3 are included in Figs 5-7 which are obtained by the Yule-Walker

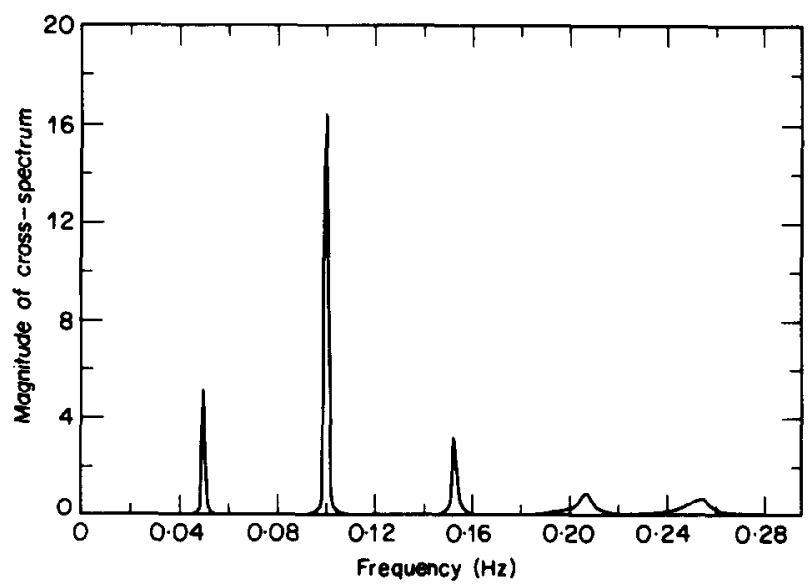

Figure 5. Cross-spectral magnitude by the Yule-Walker algorithm (filter order $=20$ ) for the simulated string.

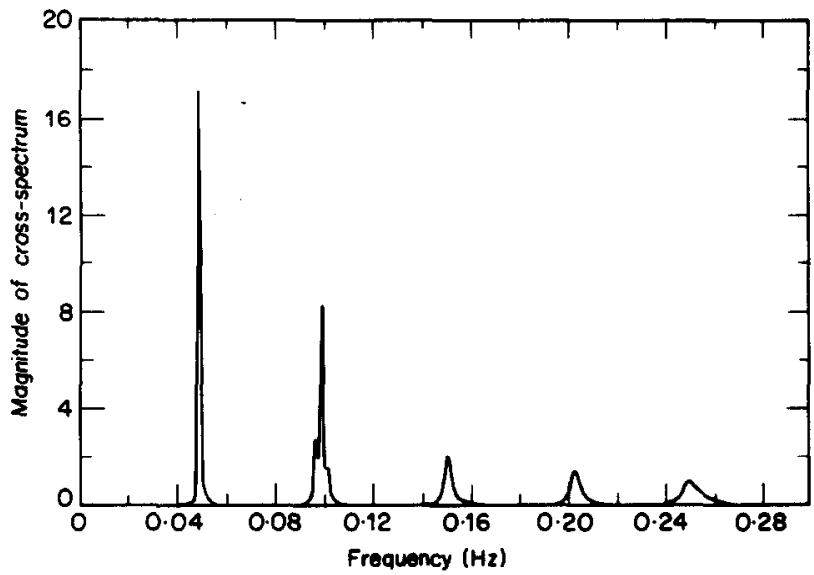

Figure 6. Cross-spectral magnitude by the Burg algorithm (filter order $=20$ ) for the simulated string. 


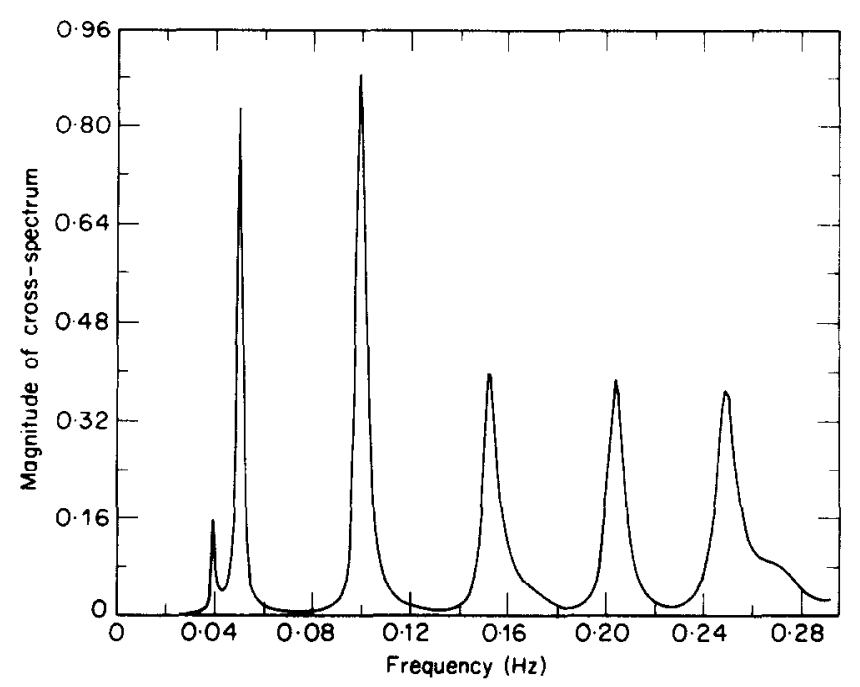

Figure 7. Cross-spectral magnitude by MLM for the simulated string.

and Burg algorithms, and MLM. The theoretical and estimated lowest three natural frequencies are listed in Table 6.

The estimated mode shapes are summarised in Table 7.

Plots of the estimated mode shapes are shown in Figs 8-10. From the above two simulations and our experiences with many measured field data, the three methods by Yule-Walker, Burg, and MLM give comparable results in both natural frequency estimation and mode shape estimation. For the modal shape identification, our cases have shown that the multichannel MLM's performance is no worse than the multichannel MEM's.

\section{SUMMARY}

In this work we have applied different algorithms of the multichannel MEM and MLM to estimate the mode shapes and compare their performances through simulations. Since many flexible parameters in the algorithms affect their performance, there is no unique way to compare their ability and draw conclusions. Our results show that both multichannel MEM and MLM are capable of estimating the mode shapes of a structure in random vibration.

TABLE 6

\begin{tabular}{llll}
\hline & \multicolumn{3}{c}{ Frequency/Hz } \\
\cline { 2 - 4 } \multicolumn{1}{c}{ Method } & Mode 1 & Mode 2 & Mode 3 \\
\hline Undamped & 0.050 & $0 \cdot 100$ & 0.150 \\
Yule-Walker & 0.050 & 0.100 & 0.153 \\
Burg & 0.0488 & 0.0989 & 0.151 \\
MLM & 0.050 & 0.100 & 0.152 \\
\hline
\end{tabular}


TABLE 7

Yule-Walker, Burg and MLM with filter order $M+1=20$ and $\alpha=\beta=0.006$

\begin{tabular}{|c|c|c|c|c|}
\hline Mass & Undamped & Yule-Walker & Burg & MLM \\
\hline \multicolumn{5}{|c|}{ First mode } \\
\hline $1 \& 2$ & $1 \cdot 902$ & 1.906 & $1 \cdot 890$ & 1.904 \\
\hline $1 \& 3$ & $2 \cdot 618$ & 2.632 & 2.605 & 2.629 \\
\hline $1 \& 4$ & $3 \cdot 078$ & $3 \cdot 102$ & 3.009 & $3 \cdot 106$ \\
\hline $1 \& 5$ & $3 \cdot 236$ & $3 \cdot 267$ & $3 \cdot 193$ & $3 \cdot 265$ \\
\hline $1 \& 6$ & 3.078 & $3 \cdot 102$ & $3 \cdot 275$ & $3 \cdot 107$ \\
\hline $1 \& 7$ & $2 \cdot 618$ & $2 \cdot 641$ & $2 \cdot 719$ & $2 \cdot 623$ \\
\hline $1 \& 8$ & 1.902 & $1 \cdot 918$ & 1.882 & 1.921 \\
\hline $1 \& 9$ & $1 \cdot 000$ & $1 \cdot 010$ & $1 \cdot 001$ & 1.004 \\
\hline \multicolumn{5}{|c|}{ Second mode } \\
\hline $1 \& 2$ & 1.618 & 1.604 & 1.591 & 1.594 \\
\hline $1 \& 3$ & 1.618 & 1.572 & $1 \cdot 551$ & 1.557 \\
\hline $1 \& 4$ & $1 \cdot 000$ & 0.914 & 0.918 & 0.905 \\
\hline $1 \& 5$ & 0.000 & $-0 \cdot 115$ & -0.010 & $-0 \cdot 103$ \\
\hline $1 \& 6$ & $-1 \cdot 000$ & $-1 \cdot 083$ & $-1 \cdot 045$ & $-1 \cdot 086$ \\
\hline $1 \& 7$ & $-1 \cdot 618$ & -1.662 & -1.661 & -1.678 \\
\hline $1 \& 8$ & -1.618 & $-1 \cdot 591$ & $-1 \cdot 649$ & -1.656 \\
\hline $1 \& 9$ & $-1 \cdot 000$ & -1.016 & -1.009 & $-1 \cdot 020$ \\
\hline \multicolumn{5}{|c|}{ Third mode } \\
\hline $1 \& 2$ & $1 \cdot 176$ & $1 \cdot 192$ & $1 \cdot 164$ & $1 \cdot 180$ \\
\hline $1 \& 3$ & $0 \cdot 382$ & 0.419 & 0.415 & 0.403 \\
\hline $1 \& 4$ & -0.727 & -0.692 & -0.712 & -0.696 \\
\hline $1 \& 5$ & $-1 \cdot 236$ & $-1 \cdot 193$ & $-1 \cdot 211$ & $-1 \cdot 208$ \\
\hline $1 \& 6$ & -0.727 & -0.717 & -0.713 & -0.712 \\
\hline $1 \& 7$ & $0 \cdot 382$ & 0.367 & 0.369 & 0.372 \\
\hline $1 \& 8$ & $1 \cdot 176$ & $1 \cdot 122$ & $1 \cdot 157$ & $1 \cdot 153$ \\
\hline $1 \& 9$ & 1.000 & $1 \cdot 028$ & 1.005 & 0.987 \\
\hline
\end{tabular}

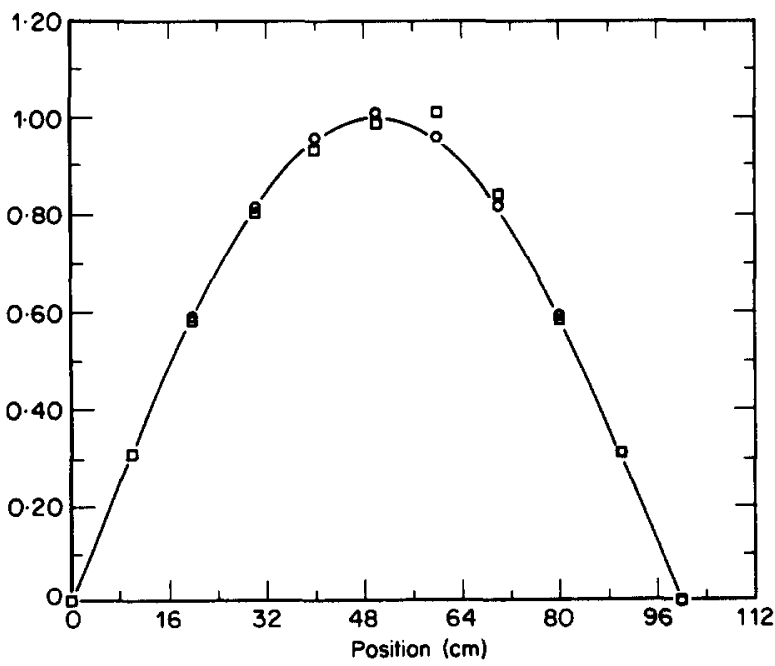

Figure 8. The estimated and theoretical first modal shape of the string. $\square$, Burg; + , MLM; $O$, Yule-Walker. 


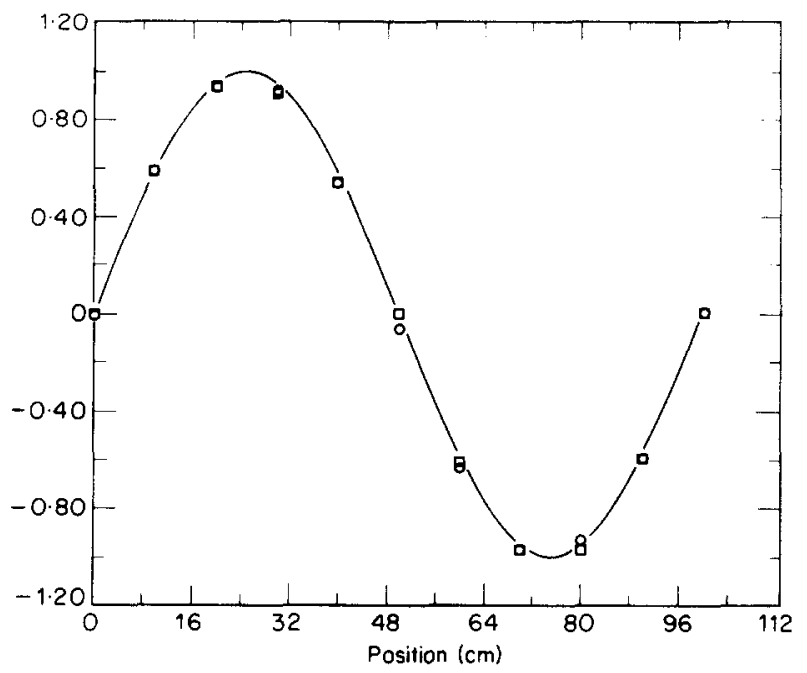

Figure 9. The estimated and theoretical second modal shape of the string. $\square$, Burg; + , MLM; $\bigcirc$, Yule-Walker.

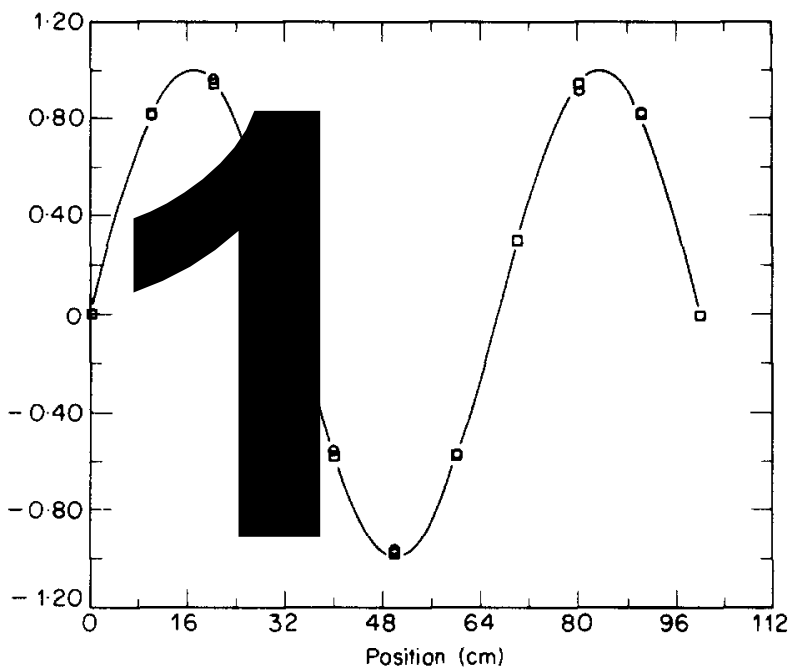

Figure 10. The estimated and theoretical third modal shape of the string. $\square$, Burg;,+ MLM; $\bigcirc$, Yule-Walker.

\section{REFERENCES}

1. J. S. Bendat and A. G. PIERSOl 1971 Random Data: Analysis and Measurement Procedures. New York: Wiley-Interscience.

2. M. J. BriggS and J. K. VANDIVER 1982 Offshore Technology Conference (OTC) 4286, Houston. Multichannel maximum entropy method of spectral analysis applied to offshore platforms.

3. B. G. BURKE and N. R. SOSDIAN 1982 Fourteenth Annual Offshore Technology Conference (OTC) 4284, pp. 625-635, Houston. Analysis of ambient vibration data by multiple shape vectors.

4. B. G. BURKe, C. SUndaraRAJAN ane F. M. SAFARI 1980 Twelfth Annual Offshore Technology Conference (OTC) 3862, pp. 63-69, Houston. Characterization of ambient vibration data by response shapes vectors. 
5. S. HAYKIN, editor 1979 Nonlinear Methods of Spectral Analysis. Topics in Applied Physics, Vol. 34. Berlin: Springer-Verlag.

6. M. A. Lagunas-Hernandes and A. Gasull-Llampallas 1984 IEEE Transactions on Acoustics, Speech, and Signal Processing ASSP-32, 170-173. An improved maximum likelihood method for power spectral density estimation.

7. M. A. Lagunas-Hernandes, M. E. Santamaria and A. Gasull-Llampallas 1985 IEEE International Conference on Acoustics, Speech, and Signal Processing (ICASSP), p. 77. Cross-spectral maximum likelihood estimate.

8. C. Y. LIOU 1985 Ph.D. Thesis, Department of Ocean Engineering, Massachusetts Institute of Technology. A separable cross-entropy approach to power spectral estimation.

9. M. MORF, A. VIEIRA, T. L. LEE and T. KAILATH 1978 IEEE Transactions of Geoscience Electronics GE-16, 85-94. Recursive multichannel maximum entropy spectral estimation.

10. A. V. OpPenheim and R. W. SChAfer 1975 Digital Signal Processing. Englewood Cliffs, NJ: Prentice-Hall.

11. J. M. SMITH 1977 Mathematical Modeling and Digital Simulation for Engineers and Scientists. New York: Wiley-Interscience.

12. O. N. STRAND 1977 IEEE Transactions on Automatic Control AC-22, 634-640. Multichannel complex maximum entropy (autoregressive) spectral analysis. 